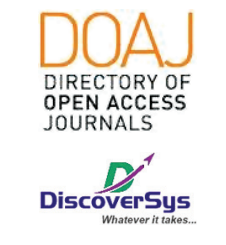

Published by DiscoverSys

\section{Potential combinations of Platelet Rich Plasma (PRP) and chitosan in burn wound management: A review}

\author{
Gede Wara Samsarga, ${ }^{*}{ }^{*}$ I Gusti Putu Hendra Sanjaya, ${ }^{1}$ \\ Agus Roy Rusly Hariantana Hamid, ${ }^{1}$ Komang Januartha Putra Pinatih, ${ }^{3}$ \\ Ni Made Ratih Purnama Dewi ${ }^{3}$
}

CrossMark

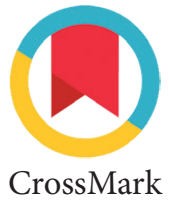

\title{
ABSTRACT
}

Background: The development of new modality for burn wound healing is necessary to reduce morbidity and mortality of burn injury. PRP (Platelet Rich Plasma) is one of potential modality for burn injury because of its ability to deliver various growth factors to initiate and accelerate tissue healing and regeneration. Clinical efficacy of PRP is frequently disturbed because inconsistency and inefficiency of growth factor release in PRP. To increase the effectiveness of PRP in wound healing and increase the bioavailability of growth factor, PRP can be combined with a carrier that can control continuous release of growth factors. Chitosan is one of biomaterial that potential to use because of its biodegradability characteristic and biological activity that important for burn wound healing and prevent the formation of scar tissue in a burn injury. Researches have shown that chitosan is an effective carrier to facilitate growth factor release continuously to burn wound.

Aims: This review aims to review potential Combinations of PRP (Platelet Rich Plasma) \& Chitosan in Burn Wound

Conclusion: With chitosan ability to facilitate burn wound healing and as an effective carrier, combination of PRP and chitosan is a promising modality for burn wound healing.
'Division of Plastic, Reconstructive and Aesthetic Surgery, Department of Surgery, Faculty of Medicine Udayana University, Bali, Indonesia ${ }^{2}$ Department of Clinical Microbiology, Faculty of Medicine, Udayana University, Bali Indonesia

${ }^{3}$ Alumni of Faculty of Medicine Udayana University, Bali, Indonesia

${ }^{*}$ Correspondence to: Gede Wara Samsarga, Division of Plastic, Reconstructive and Aesthetic Surgery, Department of Surgery, Faculty of Medicine Udayana University, Bali, Indonesia; warasamsarga@unud.ac.id

Received: 2020-03-29 Accepted: 2020-05-22 Published: 2020-07-28
Keywords: Burn Injury, PRP, Growth factor, Chitosan

Cite This Article: Samsarga, G.W., Sanjaya, I.G.P.H., Hamid, A.R.R.H., Pinatih, K.J.P., Dewi, N.M.R.P. 2020. Potential combinations of Platelet Rich Plasma (PRP) and chitosan in burn wound management: A review. Intisari Sains Medis 11(2): 571-574. D0l: 10.15562/ism.v11i2.742

\section{INTRODUCTION}

Millions of people around the world are treated in health care sites due to burns every year, and thousands of them die. ${ }^{1}$ In 2014, the World Health Organization (WHO) estimated that 265,000 deaths were occurring annually worldwide due to burns. ${ }^{1}$ 45,000 burns require treatment in hospitals in the United States each year. Of these cases, 20,000 cases were major burns involving $25 \%$ of the body surface area and 4,500 people died. ${ }^{2}$

Methods to improve the optimal healing process of wounds and tissues are needed to improve survival and quality of life in patients with burns and can also reduce costs incurred due to the treatment of burns. ${ }^{3}$ Healing on the burn wound is a complex process and involves various cell components and matrix components. Burns have a different healing process when compared to incision or excision wounds. In burns, the process of necrosis occurs progressively and can extend to deeper tissues, thereby inhibiting the reepithelization process. Because the mechanism of healing of burns is different from other injuries, the development of biomaterials for healing burns is needed to reduce the morbidity and mortality of burns. ${ }^{3}$

Platelet Rich Plasma (PRP) is an autologous product that concentrates large amounts of platelets in a small volume of blood plasma. ${ }^{4}$ PRP contains a variety of growth factors essential to initiate and accelerate tissue healing and regeneration. Platelet-Rich Plasma (PRP) is a procedure that relatively easy, low cost and minimally invasive to deliver autologous growth factors for wound healing. ${ }^{5}$ Because of these benefits, PRP is now one of the innovative modalities with excellent potential for healing both chronic and acute wounds such as in diabetic wounds, pressure sores and burns. Fundamentally the mechanism of PRP is molecular and cellular stimulation of normal wound healing, as observed in platelet activation. Platelets can be activated by two types of stimuli including physical stimuli such as heat, cold, and vibration and chemical stimuli such as collagen, lipopolysaccharides and chitosan. ${ }^{6}$

Chitosan is a polysaccharide derived from chitin which is a natural component obtained from shrimp shells or crabs. Chitosan is relatively easy and inexpensive to achieve because it comes from shrimp shell waste and is processed with relatively simple techniques. Chitosan and its oligomer are found to be able to improve wound healing, especially in the proliferation phase and extracellular matrix formation. Chitosan and its oligomer are known to have excellent biological properties so that they can be applied to various fields. Lysosomes slowly 
hydrolyze the membranes in chitosan and produce chito-oligomers which can stimulate the deposition, arrangement and orientation of collagen fibrils in extracellular matrix components. Besides, chitosan membranes are also indicated to be able to stimulate migration of inflammatory cells and induce cellular organization. ${ }^{4}$ Due to various biological properties in accelerate wound healing, the ability as a vector of the growth factors and its ability in the activation of platelets, the use of chitosan as a stimulant for platelet activation in PRP highly potential and effective to use for the healing of wounds, especially burns. ${ }^{6}$

\section{THE ROLE OF PRP IN BURN WOUND}

Platelet Rich Plasma (PRP) can be defined as blood plasma containing 1,000,000 platelets/microliters with a volume of $5 \mathrm{ml}$ of plasma. PRP contains seven types of growth factors, namely: Platelet-Derived Endothelial Cell Growth Factor-AA (PDGF-AA), PDGF-BB, PDGF-AB, Transforming Growth Factor $\beta 1$ (TGF- $\beta 1$ ), TGF- $\beta 2$, Vascular Endothelial Growth Factor (VEGF-AB), and Epidermal Growth factor (EGF).?

The mechanism of PRP in improving the wound healing process is based on the increased delivery of growth factors in the wound. ${ }^{7}$ Growth factors are known to mediate the normal wound healing process and mediate impaired wound healing processes such as in burns. ${ }^{8}$ Growth factors attract cells to the wound site and stimulate cell proliferation and increase differentiation and deposition of the extracellular matrix.

Growth factors that important in wound healing in the PRP is TGF- $\beta$ and PDGF as it affects the various phases of wound healing by triggering cell growth and differentiation. Several in vivo and in vitro studies show that all cells in healing wounds are sensitive to growth factors. ${ }^{7}$ EGF is a chemotactic factor of fibroblasts and can increase epidermal regeneration and strength of the wound. PDGF stimulates the migration and proliferation of fibroblasts, and smooth muscle cells increase collagen deposition and is a chemotactic factor for neutrophils and monocytes. TGF- $\beta 1$ regulate differentiation, proliferation and chemotaxis of cells, as well as the synthesis of a variety of extracellular matrix proteins. In animal studies, topical application of TGF- $\beta 1$ can increase collagen synthesis, granulation tissue and strength of the wound. ${ }^{7}$ TGF- $\beta$ stimulates the synthesis of collagen, bronectin, and glycosaminoglycan from fibroblasts. TGF- $\beta$ increases collagen synthesis and accelerates collagen maturation in the early period of healing wounds. Also, the use of TGF- $\beta$ PDGF together can increase collagen deposition when compared to only using TGF- $\beta{ }^{7}$

Some studies mention the effect of recombinant growth factor for the treatment of burns. ${ }^{10}$ The use of recombinant PDGF, EGF, VEGF, TGF- $\beta 2$ shows the effect of increasing and accelerating the process of healing burns in animal studies, but still requires further research in humans. The development of a combination of several growth factors in burns may be more effective when compared to using only one type of growth factor. ${ }^{10}$ PRP can be considered as a source of a combination of growth factors that are relatively inexpensive and easy to produce. ${ }^{10}$

Study-related to the use of PRP on animals showed PRP could improve vascular growth and fibroblast proliferation significantly. ${ }^{10}$ There are several mechanisms why the use of PRP in deep burns can produce benefits for healing burns. The first is the hemostatic ability of PRP to reduce perioperative blood loss and also increase the rate of skin grafts take by reducing continuous bleeding, functioning as fibrin glue, and also as an excellent vascular bed for meshed skin grafts. In addition, the positive effect of PRP on wound healing, as has been widely studied, can accelerate the closure of burns because PRP is able to increase vascular growth, fibroblast proliferation and reepithelialization. ${ }^{10}$

\section{THE ROLE OF CHITOSAN IN BURN WOUND}

Chitosan is derived from the deacetylation of chitin found in the crustacean exoskeleton. Chitosan is a linear polysaccharide composed of glucosamine and $\mathrm{N}$-acetyl glucosamine residues that combine with $\beta$-soluble glycosidic bonds. The monomer unit in chitosan has the molecular formula $\mathrm{C} 8 \mathrm{H} 12 \mathrm{NO} 5$ with levels of $\mathrm{C}, \mathrm{H}, \mathrm{N}$, and $\mathrm{O}$ respectively $47 \%, 6 \%$, $7 \%$, and $40 \%$. The biodegradable nature of chitosan has other properties including not soluble in water, organic acids, dilute and alkalies, but dissolved in concentrated acids such as nitric acid, sulfuric acid, phosphoric acid, and formic anhydrosis acid. Based on its biological and chemical properties, chitosan has a characteristic that is easily formed into sponges, gel solutions, pastes, membranes, and fibres that are useful in its use as a hemostatic agent, antimicrobial agent, and wound dressing. ${ }^{11}$

Various studies have been conducted to examine the effects of chitosan in healing wound wounds, especially in burns in experimental animals and directly in humans. These studies show chitosan can reduce the inflammatory response, increase the expression of collagen and extracellular components, and the formation of granulation tissue..11 Increased production of collagen and extracellular 
matrix shows an increase in fibroblasts, and there is an increase in the expression of several growth factors in wounds treated with chitosan. Research also shows increased re-epithelialization, increased dermo-epidermal junctions formation and increased tensile strength from wound healing. ${ }^{11}$ These things show that chitosan can affect signal transduction in the wound healing process. ${ }^{11}$

Research by Chang et al. examined the use of N-carboxymethyl chitosan on second-degree burns in mice. ${ }^{3}$ The study shows the mechanism of chitosan in wound healing is through the activation of the TGF $\beta 1$ / Smad3 pathway. ${ }^{3}$ Through this pathway, TGF $\beta 1$ can facilitate the synthesis of type I / III collagen and elastin in fibroblasts, increase fibronectin expression, decrease collagen fibre degradation and increase exudation from the extracellular matrix. ${ }^{3}$ TGF $\beta 1$ can inhibit Smad3, which is needed in wound healing and wound contraction. ${ }^{3}$ Research by Baxter et al. shows that the use of chitosan in burns is capable of producing a biphasic response of TGF $\beta 1$ in which an increase in TGF $\beta 1$ expression occurs in the initial phase of a burn followed by a decrease in TGF $\beta 1$ in the remodelling phase. ${ }^{2}$ Increased expression of the TGF $\beta 1$ gene in the early phase of wound healing is necessary to regenerate lost tissue and the production of the collagen matrix. In the remodelling phase, excessive collagen production is undesirable because it will cause scarring in burns. This shows that the use of chitosan in the regulation of gene expression TGF $\beta 1$ to biphasic collagen production is potential for improving tissue repair in the early phase of burns accompanied by a minimal scar tissue formation in the late phase of the healing of burns. ${ }^{2}$

\section{POTENTIAL COMBINATION OF CHITOSAN AND PRP IN BURN WOUND}

An essential procedure in Platelet Rich Plasma (PRP) preparation is platelet activation so that it can produce growth factors. The addition of thrombin is often given to PRP to be able to activate platelets. Still, thrombin which is commercially available today is an antigen and causes sensitivity and allergic reaction if injected into experimental animals. Because of its hemostatic activity, thrombin cannot be injected in large blood vessels because it results in intravascular freezing and ultimately causes death. Research by Shen et al. shows that chitosan is potentially developed as a substitute for thrombin in PRP. ${ }^{11}$ The study showed no significant difference between the ability of chitosan and thrombin to induce platelet activity. ${ }^{11}$ Platelets have membranes with negative charge due to the presence of negatively charged glucose groups, negative voltage phospholipids such as phosphatidylethanolamine phosphatidylcholine, carbohydrates such as sialic acid and also negative voltage amino acids such as aspartate and glutamate. Chitosan is positively charged due to the presence of free amino acid groups derived from deacetylation of $\mathrm{N}$-acetyl-Dglucosamine. Chitosan is very positive and binds strongly and attracts molecules with negative voltage so that this interaction has the potential to activate platelets. ${ }^{5}$ Activation of platelets will result in the release of various growth factors $s$ from alpha-granules. ${ }^{5}$

The clinical efficacy of PRP is often disrupted due to the delivery of inconsistent and inefficient growth factors in PRP. Growth factors are released quickly so that its activity and clinical benefit in wound healing is reduced. To improve the effectiveness of PRP on wound healing and improve the bioavailability of growth Factors, PRP can be combined with a carrier that can release growth factors continuously and in a controlled manner. ${ }^{12}$ Several studies have shown the effects of chitosan in increasing the effectiveness of PRP. ${ }^{11,12,13}$ PRP-loaded chitosan scaffolds has the potential to be developed as an effective carrier on the application of PRP to facilitate the release of growth factors continuously. ${ }^{12}$

Chitosan has been widely studied as a scaffold with biological properties that can improve the wound healing process independently. ${ }^{11,12,14}$ Other studies on the effect of chitosan as a carrier of growth factors recombinant or growth factors at PRP show prospective and significant results. ${ }^{12,14}$ Research shows the effects of chitosan as an effective conductor of growth factors where chitosan can increase the release of growth factors and can maintain the active form of growth factor $\mathrm{s}$ from platelets. ${ }^{12}$ Chitosan can provide a microenvironment that is conducive to extend the viability of cells which have the function of angiogenic and osteogenic and gel formulations of chitosan can maintain the viability of a growth factor $s$ or protein is incorporated and viability of host cells such as fibroblasts. ${ }^{12}$ In addition, chitosan is also able to extend the platelet lysates contact with the injured tissue for the right time to induce a therapeutic effect. ${ }^{14}$ Based on these benefits, chitosan is a potential and effective carrier in PRP to produce continuous-release, thereby increasing the bioavailability of growth factors for wound healing, especially burns.

\section{CONCLUSION}

Platelet Rich Plasma (PRP) is a modality that has been developed for wound healing because of its ability to deliver growth factors that are useful in the process of wound healing, especially burns. To increase the 
bioavailability of growth factors, $\mathrm{PRP}$ requires a carrier system that can deliver growth factors in a controlled and continuous manner, and this can be achieved by adding chitosan to the PRP. Further research is still needed for the right formulation and the development of a combination of chitosan and PRP not only in the process of healing wounds, especially burns, but in other regenerative fields.

\section{ACKNOWLEDGMENTS}

We are thankful to all the staff for helping with the data retrieval.

\section{CONFLICT OF INTEREST}

We declare that there were no conflicts of interest in this study.

\section{FUNDING}

The authors are responsible for the study funding without the involvement of grant, scholarship, or any other resources of funding.

\section{AUTHOR CONTRIBUTION}

All of authors are equally contributed to the study from the study framework, data gathering, data analysis, until reporting the result of study.

\section{REFERENCES}

1. World Health Organization. Burns: Fact sheets. 2014. Available from http://www.who.int/mediacentre/ factsheets/fs365/en/\#.

2. Baxter RM, Dai T, Kimball J, Wang E, Hamblin MR, Wiesmann WP et al. Chitosan dressing promotes healing in third degree burns in mice: gene expression analysis shows biphasic effects for rapid tissue regeneration and decreased fibrotic signaling. J Biomed Mater Res A. 2013 February; 101(2): 340-8.

3. Chang J, Liu W, Han B, Peng S, He B, Gu Z. Investigation of the skin repair and healing mechanism of $\mathrm{N}$-carboxymethyl chitosan in second-degree burn wounds. Wound Rep Reg 2013; 21:113-121.
4. Mohammadil R, Mehrtash M, Mehrtash M, Hassani1 N, Hassanpour A. Effect of Platelet Rich Plasma Combined with Chitosan Biodegradable Film on Full-Thickness Wound Healing in Rat Model. Bull Emerg Trauma. 2016;4(1):29-37.

5. Hattori H, Ishihara M. Feasibility of improving plateletrich plasma therapy by using chitosan with high platelet activation ability. Experimental and Therapeutic Medicine. 2017;13:1176-1180.

6. Cavallo C, Roffi A, Grigolo B, Mariani E, Pratelli L, Merli G, Kon E, Marcacci M, Filardo G. Platelet-Rich Plasma: The Choice of Activation Method Affects the Release of Bioactive Molecules. Hindawi BioMed Research International.2016;2016:1-7.

7. Everts PAM, Knape JTA, Weibrich G, Schönberger JPAM, Hoffmann J, Overdevest EP et al. Platelet-Rich Plasma and Platelet Gel: A Review. JECT. 2006;38:174-187.

8. Ozcelik U, Ekici Y, Bircan HY, Aydogan C, Turkoglu S, Ozen O, Moray G. Haberal M. Effect of Topical PlateletRich Plasma on Burn Healing After Partial-Thickness Burn Injury. Med Sci Monit, 2016; 22: 1903-1909.

9. Marck RS, Middelkoop E, Breederveld RS. Considerations on the Use of Platelet-Rich Plasma, Specifically for Burn Treatment. J Burn Care Res. 2014;35:219-227.

10. Ching YH, Sutton TL, Pierpont YN, Robson MC, Payne WG. The use of growth factors and other humoral agents to accelerate and enhance burn wound healing. Eplasty 2011;11:e41.

11. Shen EC, Chou TC, Gau CH, Tu HP, Chen YT and Fu E: Releasing growth factors from activated human platelets after chitosan stimulation: A possible bio-material for plateletrich plasma preparation. Clin Oral Implants Res 17: 572-578, 2006.

12. Kutlu B, Tiğlı Aydın RS, Akman AC, Gümüşderelioglu M and Nohutcu RM: Platelet-rich plasma-loaded chitosan scaffolds: Preparation and growth factor release kinetics. J Biomed Mater Res B Appl Biomater 101: 28-35, 2013.

13. Oktay EO, Demiralp B, Demiralp B, Senel S, Cevdet Akman A, Eratalay $\mathrm{K}$ and Akincibay $\mathrm{H}$ : Effects of platelet-rich plasma and chitosan combination on bone regeneration in experimental rabbit cranial defects. J Oral Implantol 36: 175-184, 2010.

14. Busilacchi A, Gigante A, Mattioli-Belmonte M, Manzotti S and Muzzarelli RA: Chitosan stabilizes platelet growth factors and modulates stem cell differentiation toward tissue regeneration. Carbohydr Polym 98: 665-676, 2013.

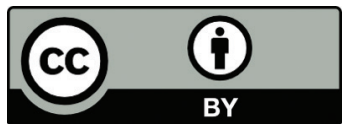

This work is licensed under a Creative Commons Attribution 